\title{
The electric field gradient asymmetry parameter in InSe
}

\author{
Z.D. Kovalyuk ${ }^{1}$, A.G. Khandozhko ${ }^{2}$, G.I. Lastivka ${ }^{2}$, A.P. Samila ${ }^{2}$ \\ ${ }^{1}$ Chernivtsi Department of the Institute of Materials Science Problems, NAS of Ukraine, \\ 5, Iryna Vilde str., 58001 Chernivtsi, Ukraine \\ ${ }^{2} Y u$. Fed'kovych Chernivtsi National University, \\ 2, Kotsyubyns'ky str., 58012 Chernivtsi, Ukraine
}

\begin{abstract}
The complex NQR spectra of ${ }^{115}$ In caused by presence of structural defects called polytypes are observed in a monocrystalline InSe sample. Multiple ratio of average frequencies of NQR spectra that correspond to four resonance transitions of quadrupole nuclii with spins $I=9 / 2$ testifies to existence of axial symmetry of the electric field gradient in In nucleus positions inside the InSe crystal structure. The latter is confirmed by the dependence of the NQR spectrum amplitude on the angle between the main crystal axis $c$ and vector of high-frequency field $\mathbf{H}_{1}$. However, presence of a residual intensity in the spectra at $c \| \mathbf{H}_{1}$ can indicate the existence of crystal blocks in bulk of the sample with weak angular misorientation.
\end{abstract}

Keywords: NQR, multiplet spectra, structural defects, polytypes.

Manuscript received 14.10.10; accepted for publication 16.03.11; published online 30.06.11.

\section{Introduction}

Indium selenide belongs to layered semiconductor materials of $\mathrm{GaS}$ group and attracts special interest of researchers. It is caused not only by a large number of physical effects related with strong anisotropy of the crystal structure but also by the possibility of their wide practical use. The crystal structure of InSe is formed in such a way that bonds inside the main layer have covalent character, while between the layers - Van-derWaals one. That is why, monoatomic layers Se-In-In-Se are formed in InSe [1].

The crystalline structure of layered semiconductor compound InSe admits presence of the axial-symmetrical gradient of electric field in the direction of the "In-In" bond (axis $c$, Fig. 1). It follows from the results of radiographical researches [2] as well as from spectra of electron paramagnetic resonance (EPR) [3] inherent to the crystal structure of GaS group compounds. Though, a direct proof of the existence of axial-symmetric electric field inside InSe layered crystals may be obtained using nuclear quadrupole resonance (NQR). First of all, the research of NQR spectra is a rather effective method to research crystal structure perfectness and local symmetry in the position of a resonance nucleus [4]. Secondly, the existence of the electric field gradient in the heavily anisotropic crystal structure of GaS, GaSe and InSe single crystals caused by quadrupole moments of ${ }^{69} \mathrm{Ga},{ }^{71} \mathrm{Ga}$ and ${ }^{115}$ In [5] enables the possibility to research electronic and crystal structures by means of NQR.
The purpose of this paper is to determine the structural defects and perfectness of a crystalline lattice in single crystals of indium selenide by studying the intendities of NQR spectral lines for ${ }^{115}$ In depending on orientation of the main crystal axis $c$ relatively to the vector of the high-frequency field $\mathbf{H}_{1}$.

\section{Experimental}

The research of NQR spectra was carried out using a spectrometer at continuous-wave operation in the radiofrequency bandwidth 10 to $45 \mathrm{MHz}$ at room temperature $T=293 \mathrm{~K}$. As a detector of NQR resonance signals, we used marginal oscillator [6]. Single crystals grown accordingly to the Bridgman method were used for these researches. Samples for measurements were cut of the cylinder part of grown ingot with the diameter 16 to $18 \mathrm{~mm}$. The length of the samples was 20 to $30 \mathrm{~mm}$. The fact that monoatomic layers are mostly located along the direction of growth facilitates detection of NQR signals. In this case, the cylindrical shape of the sample let to fill the volume of oscillatory circuit coil of spin generator (Fig. 2a); and the magnetic component of radiofrequency field $\mathbf{H}_{1}$ was directed normally to the crystal axis $c$, that is in the direction of axial symmetry of the electric field gradient (Fig. 2b).

The maximum intensity of NQR signals was obtained due to the fact that monoatomic layers of the crystal structure are directed along the axis of radiofrequency coil (Fig. 2a). 


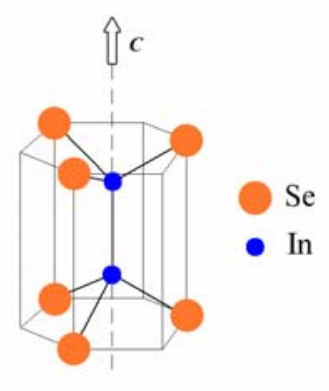

Fig. 1. Structure element of crystal lattice of InSe.

As ${ }^{115}$ In has the spin $I=9 / 2$, it means that there are four resonance transitions accordingly to selection rules: $\pm 1 / 2 \leftrightarrow \pm 3 / 2 ; \pm 3 / 2 \leftrightarrow \pm 5 / 2 ; \pm 5 / 2 \leftrightarrow \pm 7 / 2 ; \pm 7 / 2 \leftrightarrow \pm 9 / 2$. NQR frequencies $\left(v_{1}\right.$ to $\left.v_{4}\right)$ that correspond to these transitions can be obtained from the following expressions [4]:

$$
\begin{aligned}
& v_{1}=\frac{1}{24} e Q q_{z z}\left(1+9.0333 \eta^{2}-45.691 \eta^{4}\right) \\
& v_{2}=\frac{2}{24} e Q q_{z z}\left(1+1.3381 \eta^{2}-11.724 \eta^{4}\right) \\
& v_{3}=\frac{3}{24} e Q q_{z z}\left(1+0.1857 \eta^{2}-0.1233 \eta^{4}\right) \\
& v_{4}=\frac{4}{24} e Q q_{z z}\left(1+0.0809 \eta^{2}-0.0043 \eta^{4}\right)
\end{aligned}
$$

where $e$ is the charge of an electron, $Q$ - quadrupole moment of the nucleus, $q$ - electric field gradient in the direction $c$, and $\eta=\left|\frac{q_{x x}-q_{y y}}{q_{z z}}\right|$ - the electric field gradient asymmetry parameter [4]. Generally, a direction of the main axes $x, y, z$ of the tensor for the electric field gradient is chosen in such a manner that $q_{x x}<q_{y y}<q_{z z}$, and then $0<\eta<1$. When the electric field has axiallysymmetric distribution, $\eta=0$. That is why it can be eliminated from the expressions(1). Four zones of resonance frequencies with average values of 10.25, 20.5, 30.8 , and $41 \mathrm{MHz}$ that approximately satisfy the ratio $v_{1}: v_{2}: v_{3}: v_{4}=1: 2: 3: 4$ were found in InSe for ${ }^{115}$ In isotope. The latter shows insignificant asymmetry of the electric field gradient on ${ }^{115} \mathrm{In}$ and its distribution can be considered as axial-symmetric. We have researched the range of frequencies that correspond to the transition $\pm 3 / 2 \leftrightarrow \pm 5 / 2$. ${ }^{115}$ In NQR spectrum at room temperatures is located within the range of $20.4 \ldots 20.7 \mathrm{MHz}$ in this case.

NQR spectra of ${ }^{115} \mathrm{In}$ in InSe shown in Fig. 3 were recorded using the method of frequency modulation [4], therefore derivatives of the second order for spectral lines of the Gaussian shape were observed. The spectrum consists of three multiplet groups with the maximum intensity of lines at the frequencies 20.485, 20.540, and $20.595 \mathrm{MHz}$, accordingly.
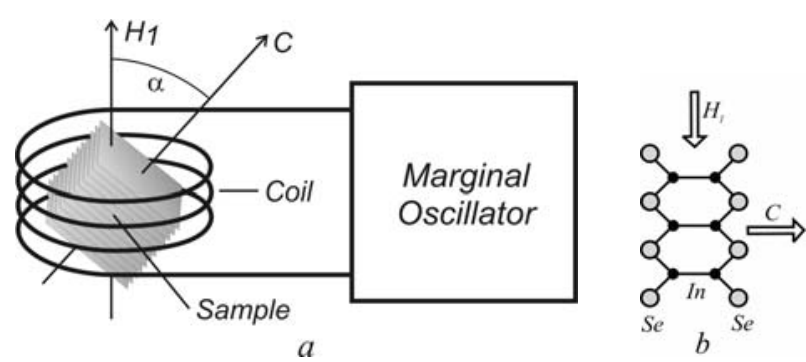

Fig. 2. Location of crystal InSe layers relatively to the vector of radio-frequency magnetic field $\mathbf{H}_{1}: a$ - location of the sample (part of the ingot) in the coil of LC oscillatory circuit circuit of the NQR spin-detector; $b$ - orientation of $\mathbf{H}_{1}$ vector relatively to crystal atomic layer.

The orientation dependence of the intensity inherent to ${ }^{115}$ In NQR at the angles $(\alpha)$ between the crystal axis $c$ and direction of actuating radio-frequency field $\mathbf{H}_{1}$ was also researched within the frequency range 20.4 to $20.7 \mathrm{MHz}$. A special device with rotary crystal enclosure was designed for performing these measurements. Crystal oriented samples with dimensions $10 \times 10 \times 10 \mathrm{~mm}$ were used for researches in this case. They were rotated in the coil spin-detector during these measurements by using the angle steps of $5^{\circ}$. Digital averaging was used for enhancement of signal-to-noise ratio.

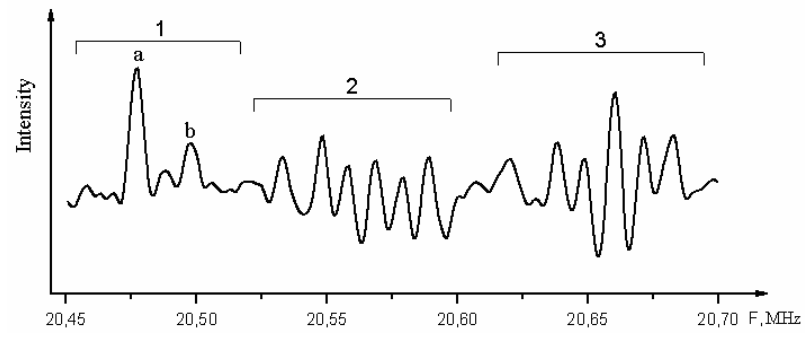

Fig. 3. ${ }^{115}$ In NQR spectrum in InSe that corresponds to the resonance transition $\pm 3 / 2 \leftrightarrow \pm 5 / 2, T=293 \mathrm{~K}$.

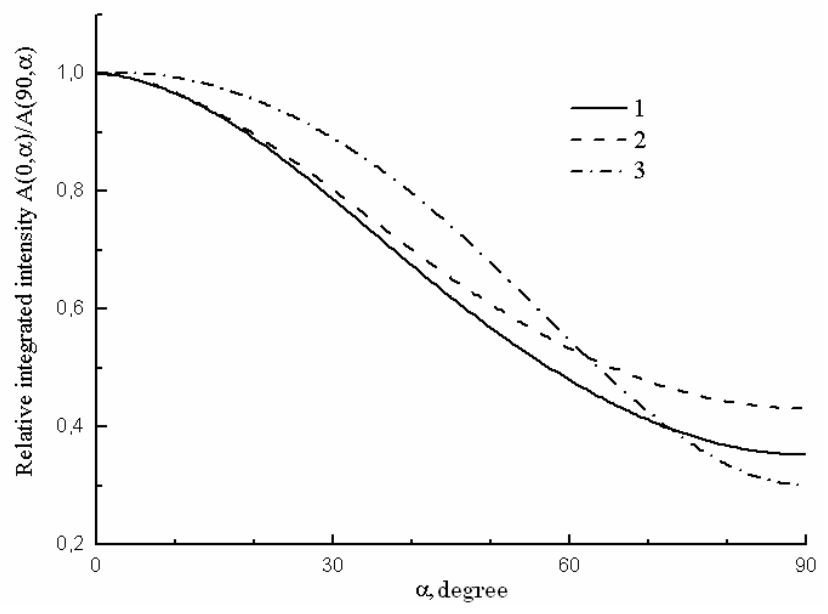

Fig. 4. Diagram of the orientation dependence of the intensity of NQR lines in InSe sample: 1 - range $20.450-20.520 \mathrm{MHz}$ 2 - range 20.510-20.600 MHz; 3 - range 20.600-20.700 MHz. 
Fig. 4 shows the diagram of dependence for the relative integrated intensity of resonance lines for these three multiplet groups in the NQR spectrum. There is a dependence of the intensity on the direction of $\mathbf{H}_{1}$ vector regarding crystallographic axis $c$ for all lines of the spectrum. The maximum of the resonance intensity really corresponds to the case when $\mathbf{H}_{1} \perp c$. If $\mathbf{H}_{1} \| c$ (that corresponds to $\alpha=90^{\circ}$, Fig. 3), the resonance intensity falls to zero for all multiplet groups " 1 ", " 2 " and " 3 ".

\section{Discussion of the results}

Observation of ${ }^{115}$ In NQR spectra in InSe in four frequency ranges with average values that satisfy the ratio $v_{1}: v_{2}: v_{3}: v_{4}=1: 2: 3: 4$ indicates insignificant asymmetry of the electric field gradient on ${ }^{115}$ In nuclii, and its distribution in the InSe crystal structure can be considered as axial-symmetric. The character of orientation dependences of ${ }^{115}$ In NQR intensity on the angle between vectors $c$ and $\mathbf{H}_{1}$ is forcible argument for this statement. Accordingly to [7], the intensity of NQR signal tends to zero in the case of axial-symmetric gradient of electric field on the nuclear when rotating the monocrystalline sample to the congruence of $c$ and $\mathbf{H}_{1}$ directions. Presence of a residual intensity of spectra at $c \| \mathbf{H}_{1}$ in our case is indicative of the existence of weak angular misorientation inherent to crystal blocks.

To check the asymmetry parameter $\eta$, the resonance frequencies of two lines in the group " 1 " for the transitions $\pm 3 / 2 \leftrightarrow \pm 5 / 2$ and $\pm 7 / 2 \leftrightarrow \pm 9 / 2$ have been measured. At room temperature $(T=293 \mathrm{~K})$ the relations of resonance frequencies $v_{4} / v_{2}$ for the specified transitions were: 2.00316 and 2.00307 (for lines of the group "1", Fig. 3). It confirms presence of an axial gradient of electric field near indium nuclii within errors of frequency measurements $\left( \pm 10^{-5}\right)$.

It is possible to explain the obtained results by peculiarities of InSe layered compound. In the paper [2], technology of growing the layered single crystals GaS, GaSe and InSe was described in details, and also influence of different growth methods on the crystal structure was shown. It was established that the considerable role in formation of polytypes is played by dislocations. The special attention was given in this work to researching the defects of packing by using electron microscopy and X-ray diffraction. Using the latter, it was shown that the crystal InSe possesses a very low energy of packing defects. Therefore, in this relation it is necessary to expect occurrence of polytypes when crystal is grown in non-equilibrium conditions, that is by melting methods.

A multiplet character of lines for all resonance transitions indicated the existence of a set of polytype elements in this compound. Accordingly to $[2,8]$, usually there is a phase $\operatorname{In}_{6} \mathrm{Se}_{7}$ in InSe. It is the existence of non-equivalent (in crystal sense) positions of In atoms that causes formation of separate groups of lines multiplets (groups " 2 " and "3"), in our opinion. But creation of the latter group of lines is associated with existence of quasi-disproportionate periodical distortion of the crystal grating [8].

Complicated but simply ordered character of ${ }^{115} \mathrm{In}$ NQR spectra in InSe is conditioned by the existence of peculiarities of structural defects that cannot be identified by other methods.

The performed research of NQR in InSe confirms complexity of the problem of polymorphism in layered crystals, which requires further investigations by using different experimental methods.

\section{Conclusions}

The following conclusions can be drawn on the basis of these researches.

1. The existence of multiplet ${ }^{115}$ In NQR spectra in InSe indicates the complex polytype structure of layered crystals of this compound.

2. The ratio of NQR resonance frequencies inherent to quadrupole transitions of ${ }^{115}$ In nuclii with the spin $\boldsymbol{I}=9 / 2$ and orientation dependence of the line intensity on the direction of the high-frequency field vector relatively to the main crystal axis confirm axial symmetry of the electric field gradient in InSe.

3. Presence of the residual intensity of spectra for $c \| \mathbf{H}_{1}$ is indicative of the existence of crystal blocks with weak angular misorientation in bulk of the sample. In this case, NQR can be used for the control of monocrystallinity of the samples grown up.

\section{References}

1. M.S. Brodyn and I.V. Blonskyy, Excitonic Processes in Layered Crystals. Naukova Dumka, Kyiv, 1986 (in Ukraine).

2. J.C.J.M. Terhell, Polytypism in the III-VI layer compounds // Progr. Cryst. Growth and Characterization of Polytype Struct. 7, p. 55-110 (1983).

3. V.I. Konovalov, S.S. Ishchenko, and S.M. Okulov, Axial crystal field and EPR in GaSe // Fizika tverdogo tela 6, p. 1842-1844 (1980), in Russian.

4. V.S. Grechishkin, Nuclear Quadrupole Interactions in Solids. Nauka, Moscow, 1973 (in Russian).

5. M.V. Vlasova, N.G. Kakazey, M. Kalinichenko, A.S. Litovchenko, Radiospectroscopic Properties of Inorganic Materials (Reference book). Naukova Dumka, Kiev, 1987 (in Ukraine).

6. V.V. Brailovskyi, A.P. Samila, A.G. Khandozhko, The marginal oscillator NQR \& NMR signals sensor // Sensor Electronics and Microsystem Technologies, 1, p. 20-23 (2010), in Ukrainian.

7. R. Ofer, A. Keren, Nutation versus angular dependent NQR spectroscopy and the impact of underdoping on charge inhomogeneities in $\mathrm{YBa}_{2} \mathrm{Cu}_{3} \mathrm{O}_{\mathrm{y}} / /$ Phys. Rev. B: Condens. Matter and Mater. Phys. 80, 224521 (2009).

8. T.J. Bastow, I.D. Cambell, H.J. Whitfeld, A ${ }^{69} \mathrm{Ga}$, ${ }^{115}$ In NQR study of polytypes of $\mathrm{GaS}, \mathrm{GaSe}$ and InSe // Solid State Communs. 39, p. 307-311 (1981). 